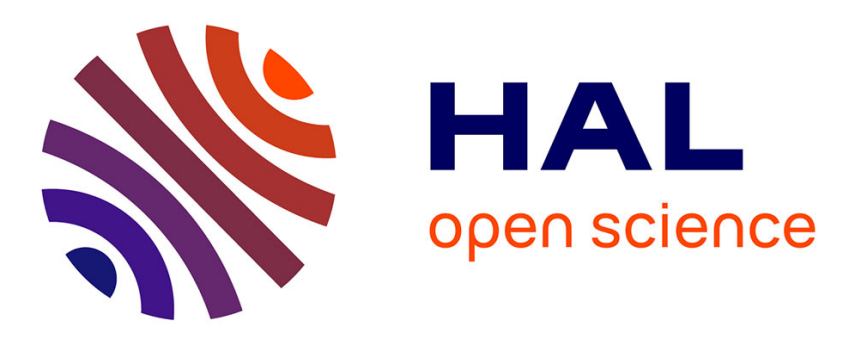

\title{
Interaction of three-level atoms with stochastic fields
}

Mohamed A. Bouchène, A. Débarre, J.-C. Keller, J.-L. Le Gouët, P. Tchénio, V. Finkelstein, P. Berman

\section{To cite this version:}

Mohamed A. Bouchène, A. Débarre, J.-C. Keller, J.-L. Le Gouët, P. Tchénio, et al.. Interaction of three-level atoms with stochastic fields. Journal de Physique II, 1992, 2 (4), pp.621-629. 10.1051/jp2:1992151 . jpa-00247661

\section{HAL Id: jpa-00247661 https://hal.science/jpa-00247661}

Submitted on 1 Jan 1992

HAL is a multi-disciplinary open access archive for the deposit and dissemination of scientific research documents, whether they are published or not. The documents may come from teaching and research institutions in France or abroad, or from public or private research centers.
L'archive ouverte pluridisciplinaire HAL, est destinée au dépôt et à la diffusion de documents scientifiques de niveau recherche, publiés ou non, émanant des établissements d'enseignement et de recherche français ou étrangers, des laboratoires publics ou privés. 
Classification

Physics Abstracts

$42.55-42.60-32.90$

\title{
Interaction of three-level atoms with stochastic fields
}

\author{
M. A. Bouchene ( $\left.{ }^{1}\right)$, A. Débarre $\left({ }^{1}\right)$, J.-C. Keller $\left({ }^{1}\right)$, J.-L. Le Gouët $\left(^{1}\right)$, \\ P. Tchénio $\left({ }^{1}\right)$, V. Finkelstein $\left({ }^{2}\right)$ and P. R. Berman $\left({ }^{2}\right)$ \\ ( $\left.{ }^{1}\right)$ Laboratoire Aimé Cotton, Bâtiment 505, 91405 Orsay Cedex, France \\ ${ }^{2}$ ) Physics Department, New York University, 4 Washington Place, New York, NY 10003, \\ U.S.A.
}

(Received 30 October 1991, accepted 10 January 1992)

\begin{abstract}
Three broadband laser pulses are used to drive transitions between three levels in $\mathrm{Sr}$ vapor. A four-wave mixing signal is monitored as a function of the delay time $t_{12}$ between two of the pulses. For sufficiently strong fields, it is found that the signal, as a function of $t_{12}$, can possess a dip or a spike which is narrower than the correlation time of the radiation fields.

A theoretical interpretation of the results is presented in terms of the so-called dark resonance.
\end{abstract}

\section{Introduction.}

In the past, much effort was devoted to determine time dependent excitation probabilities and laser-induced resonance fluorescence spectra in atomic vapors driven by a broadband field [1]. We have recently developed a coherent transient approach to investigate the interaction of a stochastic electromagnetic field with a gas of two-level atoms. In this way, new features were exposed, related either to the structure of the excitation field or to the nature of the detected quantity $[2,3]$. In the present paper, by extending our research to three-level atoms, we explore some aspects of the dark resonance effect which are revealed in a coherent transient experiment.

When two laser fields interact resonantly with a three-level folded ( $\Lambda$ or $V$ ) atomic system, the so-called dark resonance effect can occur. It corresponds to the existence of a linear combination of the atomic states which is immune to excitation by the combined laser fields $[4,5]$. When excitation is provided by non-monochromatic lasers, the isolation of the dark state crucially depends on the cross-correlation between the two light sources [6]. In the present work, excitation is provided by two totally correlated broadband fields, with an adjustable delay between them. As a function of this time-delay, the recorded signals exhibit sharp structures, much narrower than the coherence time of the light.

In either linear or non-linear optics, it is usual to record a signal as a function of a time delay between two optical paths. Those recordings often exhibit narrow structures such as the coherent artifact in pump-probe experiments [7], the coherence spike in frequency upconversion [8], or the fourth-order quantum dip in two-photon interference [9]. However, the width of those structures is never smaller than the coherence time of the light, 
$\tau_{c}$. Our unexpected observation of very narrow spikes and dips is consistent with a theoretical model that is developed within the frame of the dark resonance picture.

\section{Dark resonance.}

In the vapor that we consider, each atom has three internal states $|0\rangle,|1\rangle$ and |2) (Fig. 1). The sample is illuminated by a sequence of two fluctuating correlated pulses, labeled 1 and 2 , which are tuned to resonance with the transitions $|0\rangle-|1\rangle$ and $|0\rangle-|2\rangle$ respectively. The coherence time $\tau_{c}$ is assumed to be much smaller than the pulse duration $\tau_{L}$. The two pulses are obtained by splitting a single laser beam and they synchronously excite the sample, save for a small adjustable time delay $t_{12} \ll \tau_{L}$. The coupling of a stochastic field $\varepsilon(t) \cos [\omega t-\mathbf{k} \cdot \mathbf{r}+\phi(t)]$ with an optical transition is characterized by the Rabi frequency $\Omega(t)=\varepsilon(t) \mathrm{e}^{i \phi(t)} \mu h^{-1}$ where $\mu$ is the dipole moment of the transition. The effective interaction rate is defined as $\alpha=\left\langle|\Omega(t)|^{2}\right\rangle \tau_{c}$, where the brackets denote a

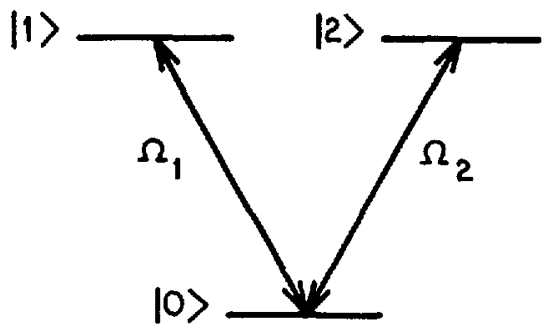

Fig. 1. - Three level configuration considered in this paper: V-system.

statistical average. A saturation parameter $S$ is defined by $S=\int \alpha \mathrm{d} t$. The quantities $S$ and $\alpha \tau_{c}$ are the effective number of interactions during the pulse duration and the light coherence time, respectively. The field is strong or weak depending upon whether $S$ is much larger or smaller than unity. In the present experiment, the first two fields $\Omega_{1}$ and $\Omega_{2}$ drive transitions $|0\rangle \Rightarrow|1\rangle$ and $|0\rangle \Rightarrow|2\rangle$ with rates $\alpha_{1}$ and $\alpha_{2}$, respectively. The corresponding saturation parameters are denoted $S_{1}$ and $S_{2}$.

When the sample is synchronously (i.e. $t_{12}=0$ ) illuminated by the fields $\Omega_{1}$ and $\Omega_{2}$, the state $|d\rangle=\cos \beta|1\rangle-\sin \beta|2\rangle$, where $\operatorname{tg} \beta=\left(\alpha_{1} / \alpha_{2}\right)^{1 / 2}$, is totally decoupled from the ground state and thus can be called a dark state [10]. In this limit, state $|c\rangle=\sin \beta|1\rangle+\cos \beta|2\rangle$ is strongly coupled to the ground state, the coupling strength being equal to $\sqrt{\Omega_{2}^{2}+\Omega_{1}^{2}}$. If there is initially no atom in states $|1\rangle$ and $|2\rangle$, the dark state, $|d\rangle$, is not populated during the pulses.

The vanishing population of $|d\rangle$ can be detected by probing the Raman coherence $\rho_{12}$ which has been built by the first two pulses between states $|1\rangle$ and $|2\rangle$. This is accomplished by a third weak pulse which irradiates the sample after the first two are terminated. The resulting four-wave-mixing signal field is proportional to $\rho_{12}\left(\tau_{\mathrm{L}}\right)$. Detection of the average signal intensity or amplitude gives access to $\left\langle\rho_{12}\left(\tau_{L}\right) \rho_{12}^{*}\left(\tau_{L}\right)\right\rangle$ or $\left\langle\rho_{12}\left(\tau_{\mathrm{L}}\right)\right\rangle$, respectively. We assume the spontaneous decay to be negligible on the time scale of the experiment. Then, the density matrix elements $\rho_{12}, \rho_{11}, \rho_{22}$ can be expressed in terms of the probability amplitudes $a_{1}$ and $a_{2}$ in states $|1\rangle$ and $|2\rangle$ as: $\rho_{12}=a_{1}^{*} a_{2}$, $\rho_{11}=n_{1}=\left|a_{1}\right|^{2}, \rho_{22}=n_{2}=\left|a_{2}\right|^{2}$. Thus, the following identity holds :

$$
\left\langle\rho_{21}\left(\tau_{\mathrm{L}}\right) \rho_{21}^{*}\left(\tau_{\mathrm{L}}\right)\right\rangle=\left\langle n_{1}\left(\tau_{\mathrm{L}}\right) n_{2}\left(\tau_{\mathrm{L}}\right)\right\rangle
$$


where $n_{1}$ and $n_{2}$ denote the populations of the states $|1\rangle$ and $|2\rangle$.

Those quantities are simply related to the dark state population in two cases, which are investigated experimentally :

i) $\alpha_{1} \ll \alpha_{2}$. Then $\beta \ll 1$ and $|d\rangle \cong|1\rangle,|c\rangle \cong|2\rangle$, so that : $\left\langle n_{1}\left(\tau_{\mathrm{L}}\right) n_{2}\left(\tau_{\mathrm{L}}\right)\right\rangle \cong$ $\left\langle n_{\mathrm{c}}\left(\tau_{\mathrm{L}}\right) n_{\mathrm{d}}\left(\tau_{\mathrm{L}}\right)\right\rangle$.

ii) $\alpha_{1}=\alpha_{2}$. Then $|d\rangle=(|1\rangle-|2\rangle) / \sqrt{2},|c\rangle=(|1\rangle+|2\rangle) / \sqrt{2}$, and $\left\langle\rho_{12}\right\rangle=$ $\left\langle n_{\mathrm{c}}-n_{\mathrm{d}}\right\rangle / 2$.

Our goal is to monitor the dark state population as the delay $t_{12}$ between the excitation pulses increases. We expect that when $t_{12}$ departs from zero, state $|d\rangle$ is no longer decoupled from other states, and there is increasing leakage of population to the dark state. When $\alpha_{1} \ll \alpha_{2}$, there is a domain of variation of $t_{12}$ over which $n_{1}$ remains much smaller than $n_{2}$. Over that domain, the rate of variation of $n_{1}$ is also much smaller than that of $n_{2}$ and the following approximation is valid:

$$
\left\langle n_{1}(t) n_{2}(t)\right\rangle \cong\left\langle n_{1}(t)\right\rangle\left\langle n_{2}(t)\right\rangle \cong\left\langle n_{\mathrm{c}}(t)\right\rangle\left\langle n_{d}(t)\right\rangle .
$$

Thus, in the two cases that we consider, the signal can be expressed in terms of averaged populations, $\left\langle\mathrm{n}_{\mathrm{c}}\left(\tau_{\mathrm{L}}\right)\right\rangle$ and $\left\langle\mathrm{n}_{\mathrm{d}}\left(\tau_{\mathrm{L}}\right)\right\rangle$.

\section{A time-delayed four-wave mixing experiment.}

In the experiment, the role of the three-level systems is played by Strontium atoms which are excited on the $5 \mathrm{~s}^{2} \mathrm{~S}_{0}-5 \mathrm{~s} 5 \mathrm{p}{ }^{3} \mathrm{P}_{1}$ transition at $\lambda=689 \mathrm{~nm}$. The degenerate upper level behaves

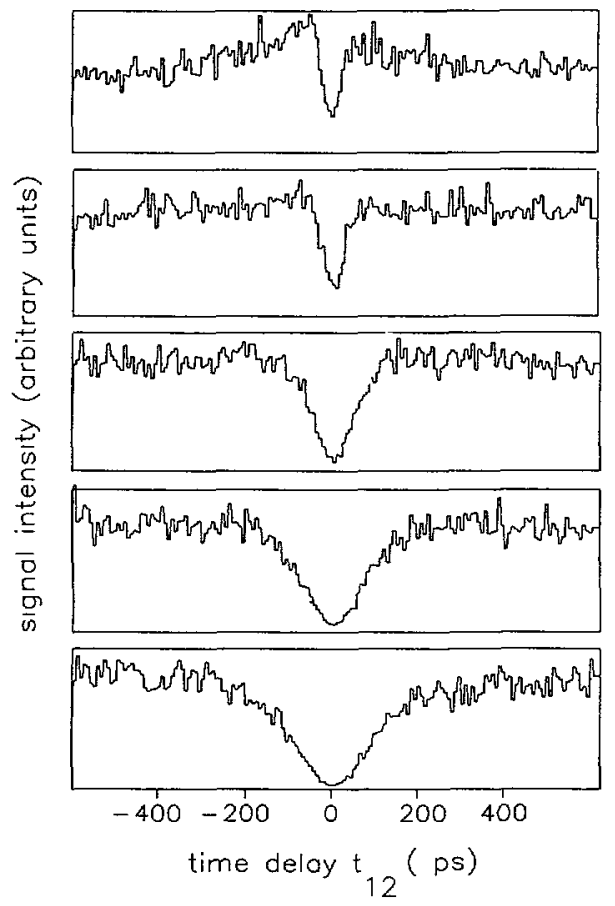

Fig. 2. - Experimental data : signal intensity as a function of the delay between the first two excitation pulses. $S_{2}=52$ and $S_{1}=17$ (a), 8.75 (b), 1.75 (c), 0.625 (d), 0.0625 (e). 


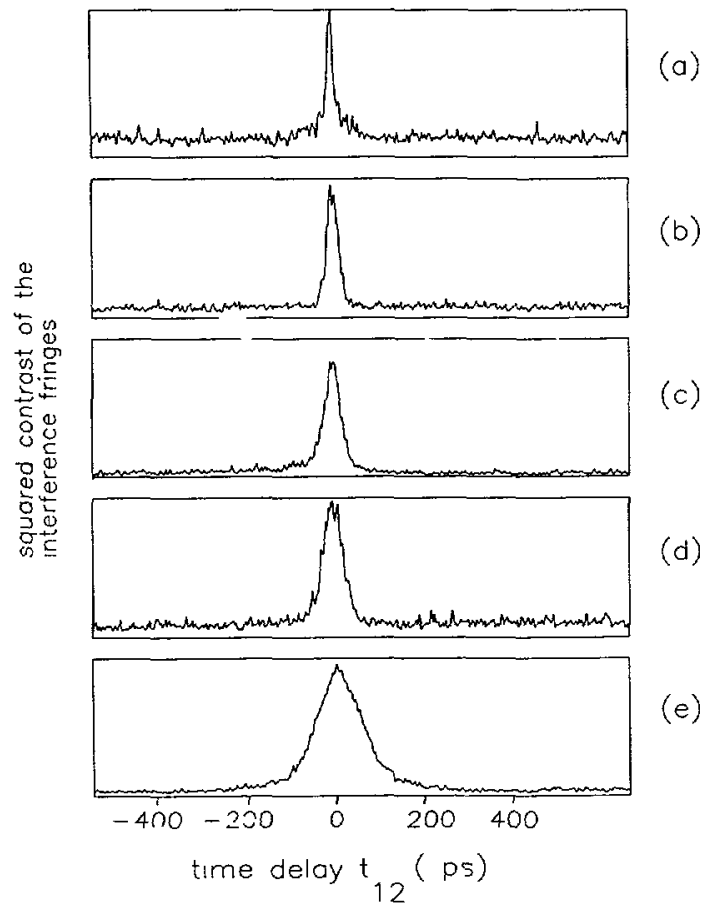

Fig. 3. - An interference pattern is formed by the signal and the reference. The squared contrast of the interference fringes is recorded as a function of the delay $t_{12}$. Those recordings are displayed in the frames (a) to (d) which correspond to the following values of the saturation parameter $S=S_{1}=S_{2}$ : 50 (a), 40 (b), 29 (c), 12 (d). Those traces reflect the variation of $\left\langle\rho_{12}\right\rangle^{2}$ as a function of $t_{12}$. The squared autocorrelation function of the field is represented in the frame (e).

as a doublet of states $|1\rangle$ and $|2\rangle$ which are selectively driven by the cross-polarized first two pulses. Laser beams 1, 2 and 3 propagate along wave vectors $\mathbf{k}_{1}, \mathbf{k}_{2}$, and $\mathbf{k}_{3}$ respectively, which are nearly colinear. The signal is detected in direction $\mathbf{k}_{3}+\mathbf{k}_{2}-\mathbf{k}_{1}$. Its polarization coincides with that of pulse 2 , which is crossed with that of pulses 1 and 3 . The pulse duration is $\tau_{\mathrm{L}} \cong 10 \mathrm{~ns}$ and the coherence time is measured to be $\tau_{\mathrm{c}} \cong 120 \mathrm{ps}$. The optical dipole lifetime is much larger than $\tau_{L}$. The first two pulses are correlated since they are beam-split from a single laser beam. For easier interpretation of the experimental results, a limitation on the field strength is imposed in order to comply with the short correlation-time requirement $\alpha \tau_{\mathrm{c}} \ll 1$.

We have noticed that, for monitoring the dark state population, it is appropriate to detect either the signal intensity or the signal amplitude, depending upon whether $\alpha_{1} \ll \alpha_{2}$ or $\alpha_{1}=\alpha_{2}$. The signal intensity is simply detected on a photomultiplier. As for the signal amplitude detection, we use a field cross-correlator device that has been described previously [11]. The signal and a synchronous replica of the probe pulse are directed to a photodiode array detector where they build an interference pattern. The squared fringe contrast is detected. It can be proven to be proportional to $\left\langle\rho_{12}\right\rangle^{2}$. Either signal is recorded as a function of the delay $t_{12}$ between the first two pulses.

Experimental profiles are displayed in figures 2 and 3 . For unbalanced excitation conditions $\left(\alpha_{1} \ll \alpha_{2}\right)$, one detects the signal intensity which is proportional to $\left\langle n_{\mathrm{c}}\left(\tau_{\mathrm{L}}\right)\right\rangle\left\langle n_{\mathrm{d}}\left(\tau_{\mathrm{L}}\right)\right\rangle$. Then the recordings exhibit a dip located around $t_{12}=0$. The signal minimum at $t_{12}=0$ 
corresponds to the absence of population in the dark state, which occurs when the first two pulses simultaneously illuminate the sample. As $t_{12}$ departs from 0 , the dark state is no longer immune to excitation and the signal intensity increases. Several profiles are obtained for different values of the field strength of pulse 1 . That of pulse 2 is not varied and the above defined saturation parameter $S_{2}$ is much larger than unity. When pulse 1 is weak, the width of the dip coincides with the coherence time $\tau_{c}$. As the intensity of pulse 1 is increased, while remaining much smaller than that of pulse 2 , the dip width becomes smaller than the coherence time $\tau_{c}$. Under balanced excitation conditions $\left(\alpha_{1}=\alpha_{2}\right)$, one detects the signal amplitude which is proportional to $\left\langle n_{\mathrm{c}}\right\rangle-\left\langle n_{\mathrm{d}}\right\rangle$. Then the recordings present a peak located at $t_{12}=0$. Indeed, at $t_{12}=0$, state $|c\rangle$ is excited while $|d\rangle$ remains unpopulated. As $t_{12}$ departs from 0 , the population difference between the two states tends to cancel. The width of the peak gets narrower as the field strength is increased. These features are predicted by the following theoretical model.

4.1 EQUATIONS OF MOTION FOR THE FIRST-ORDER STATISTICAL MOMENTS. - The quantities to be determined are the statistical averages of the populations of the excited states, in the dark state representation. This calculation is detailed in reference [10].

For sake of simplicity, we do not take account of the angles between the laser beams. We also neglect the atomic translational motion. If the Doppler phase of the atomic dipoles remains much smaller than unity on the time scale of $t_{12}$, the conclusions to be reached below are not modified by the inclusion of atomic motion. With these simplifying assumptions the density matrix equation reads :

$$
\begin{aligned}
& \dot{\rho}_{02}=-\frac{i}{2}\left(\Omega_{2}^{*}\left(n_{0}-n_{2}\right)+\Omega_{1}^{*} \rho_{12}\right) \\
& \dot{\rho}_{10}= \frac{i}{2}\left(\Omega_{1}\left(n_{0}-n_{1}\right)+\Omega_{2} \rho_{12}\right) \\
& \dot{\rho}_{12}=-\frac{i}{2}\left(\Omega_{2}^{*} \rho_{10}-\Omega_{1} \rho_{02}\right) \\
& \dot{n}_{1}=-\frac{i}{2}\left(\Omega_{1}^{*} \rho_{10}-\Omega_{1} \rho_{01}\right) \\
& \dot{n}_{2}=-\frac{i}{2}\left(\Omega_{2}^{*} \rho_{20}-\Omega_{2} \rho_{02}\right) \\
& n_{0}+n_{1}+n_{2}=N
\end{aligned}
$$

where $\rho_{02}$ and $\rho_{01}$ represent the coherences between the states linked by the optical transitions. The first two equations are formally integrated and the resulting expressions are substituted in equations for $\rho_{12}, n_{1}$, and $n_{2}$. For instance, the equation for the statistical average of $\rho_{12}$ becomes :

$$
\begin{array}{r}
\left\langle\dot{\rho}_{12}\right\rangle=\frac{1}{4} \int^{t} \mathrm{~d} t^{\prime}\left(\left\langle\Omega_{2}^{*}(t) \Omega_{1}\left(t^{\prime}\right)\left(n_{0}-n_{1}\right)\right\rangle+\left\langle\Omega_{2}^{*}\left(t^{\prime}\right) \Omega_{1}(t)\left(n_{0}-n_{2}\right)\right\rangle-\right. \\
\left.-\left\langle\Omega_{2}^{*}(t) \Omega_{2}\left(t^{\prime}\right) \rho_{12}\right\rangle-\left\langle\Omega_{1}^{*}(t) \Omega_{1}\left(t^{\prime}\right) \rho_{12}\right\rangle\right) .
\end{array}
$$

The short correlation-time requirement, $\alpha \tau_{\mathbf{c}} \ll 1$, is assumed to be satisfied. As a result atomic quantities evolve slowly on the time scale of the field fluctuations and their variations 
can be regarded as non correlated with those of the fields. Thus, the decorrelation approximation applies [12] :

$$
\left\langle\Omega_{i}^{*}(t) \Omega_{j}\left(t^{\prime}\right) A\left(t^{\prime}\right)\right\rangle \cong\left\langle\Omega_{i}^{*}(t) \Omega_{j}\left(t^{\prime}\right)\right\rangle\left\langle A\left(t^{\prime}\right)\right\rangle
$$

where $A(t)$ represents any of the atomic variables $n_{1}, n_{2}, \rho_{12}$. As a consequence, without further specification of the statistical properties of the field, all the atomic statistical moments can be expressed in terms of two-field correlation functions. The field autocorrelation function is defined by : $g(\tau)=\left\langle\Omega(t) \Omega^{*}(t+\tau)\right\rangle /\left\langle|\Omega(t)|^{2}\right\rangle$. The integral $\int_{0}^{\infty} g(\tau) \mathrm{d} \tau$ gives the coherence time $\tau_{c}$ of the light. Since $\Omega_{2}$ is the delayed replica of $\Omega_{1}$, the crosscorrelation function is given by :

$$
\left\langle\Omega_{1}^{*}(t) \Omega_{2}(t+\tau)\right\rangle=\sqrt{\alpha_{1} \alpha_{2}} g\left(\tau-t_{12}\right) / \tau_{\mathrm{c}} .
$$

One finally obtains :

$$
\begin{aligned}
\left\langle\dot{\rho}_{12}\right\rangle & =-\frac{1}{4}\left(\alpha_{1}+\alpha_{2}\right)\left\langle\rho_{12}\right\rangle+\frac{1}{4} \sqrt{\alpha_{1} \alpha_{2}}\left((1-G)\left\langle n_{0}-n_{2}\right\rangle+(1+G)\left\langle n_{0}-n_{1}\right\rangle\right) \\
\left\langle\dot{n}_{0}-\dot{n}_{1}\right\rangle & =-\alpha_{1}\left\langle n_{0}-n_{1}\right\rangle+\frac{1}{2} \sqrt{\alpha_{1} \alpha_{2}}(2-G)\left\langle\rho_{12}\right\rangle-\frac{1}{2} \alpha_{2}\left\langle n_{0}-n_{2}\right\rangle \\
\left\langle\dot{n}_{0}-\dot{n}_{2}\right\rangle & =-\alpha_{2}\left\langle n_{0}-n_{2}\right\rangle+\frac{1}{2} \sqrt{\alpha_{1} \alpha_{2}}(2+G)\left\langle\rho_{12}\right\rangle-\frac{1}{2} \alpha_{1}\left\langle n_{0}-n_{1}\right\rangle
\end{aligned}
$$

where $G=\int_{0}^{t_{12}} g(t) \mathrm{d} t / \tau_{\mathrm{c}}$, with the initial condition : $n_{0}=N, \rho_{12}=n_{1}=n_{2}=0$. In the dark state representation, the equations of motion are changed into:

$$
\begin{aligned}
\left\langle\dot{n}_{0}-\dot{n}_{\mathrm{d}}\right\rangle & =-\frac{\sqrt{\alpha_{1} \alpha_{2}}}{2} G\left\langle\rho_{\mathrm{cd}}\right\rangle-\frac{\alpha_{1}+\alpha_{2}}{2}\left\langle n_{0}-n_{\mathrm{c}}\right\rangle \\
\left\langle\dot{\rho}_{\mathrm{cd}}\right\rangle & =\frac{\sqrt{\alpha_{1} \alpha_{2}}}{4} G\left\langle n_{\mathrm{c}}-n_{\mathrm{d}}\right\rangle-\frac{\alpha_{1}+\alpha_{2}}{4}\left\langle\rho_{c d}\right\rangle \\
\left\langle\dot{n}_{0}-\dot{n}_{\mathrm{c}}\right\rangle & =\frac{\sqrt{\alpha_{1} \alpha_{2}}}{2} G\left\langle\rho_{\mathrm{cd}}\right\rangle-\left(\alpha_{1}+\alpha_{2}\right)\left\langle n_{0}-n_{\mathrm{c}}\right\rangle
\end{aligned}
$$

The motion described by these equations clearly takes place on two different time scales which are characterized by the rate coefficients $\sqrt{\alpha_{1} \alpha_{2}} G$ and $\alpha_{1}+\alpha_{2}$. Equations are solved easily when fast and slow components can be identified, a situation that is realized when $S_{1}+S_{2} \gg 1$, and $G \sqrt{\alpha_{1} \alpha_{2}} \ll \alpha_{1}+\alpha_{2}$. The former condition is satisfied when at least one of the driving fields is strong. The latter one is fulfilled either if the field intensities are very different, or if the two laser pulses nearly synchronously excite the sample (i.e. $\left.t_{12} \ll \tau_{c}\right)$. Then, after an initial transient step of duration $\left(\alpha_{1}+\alpha_{2}\right)^{-1}$, the two levels $|0\rangle$ and $|c\rangle$, which are linked by strongly driven transition, achieve equal populations, while the population of level d is still negligible to first order in $G \operatorname{tg} \beta$. For $t>\left(\alpha_{1}+\alpha_{2}\right)^{-1}$ the rate of time variation of the density matrix elements in equations (8) is much smaller than $\alpha_{1}+\alpha_{2}$. Thus equations $(8 \mathrm{~b}, \mathrm{c})$ can be considered as stationary and they are solved for $\left\langle\rho_{\mathrm{cd}}\right\rangle$ and $\left\langle n_{0}-n_{\mathrm{c}}\right\rangle$, which are expressed in terms of $\left\langle n_{\mathrm{c}}-n_{\mathrm{d}}\right\rangle$. Those quantities are substituted into equation $(8 \mathrm{a})$, which finally reads :

$$
\left\langle\dot{n}_{\mathrm{c}}-\dot{n}_{\mathrm{d}}\right\rangle=-\frac{3}{4} \frac{G^{2} \alpha_{1} \alpha_{2}}{\left(\alpha_{1}+\alpha_{2}\right)}\left\langle n_{\mathrm{c}}-n_{\mathrm{d}}\right\rangle .
$$


It results that, after extinction of the first two pulses, the level populations are given by :

$$
\begin{aligned}
\left\langle n_{\mathrm{d}}\right\rangle & =\frac{N}{3}\left(1-\exp -\frac{3}{4} S_{1} G^{2} \cos ^{2} \beta\right) \\
\left\langle n_{\mathrm{c}}-n_{\mathrm{d}}\right\rangle & =\frac{N}{2} \exp -\frac{3}{4} S_{1} G^{2} \cos ^{2} \beta .
\end{aligned}
$$

As expected, state $|\mathrm{d}\rangle$ is not populated at $t_{12}=0$. Its population increases as $t_{12}$ departs from 0 , reflecting the breakdown of the dark state immunity to excitation.

4.2 Discussion. - The main features in the experimental profiles are consistent with the result of this calculation. The two cases of unbalanced and balanced excitation have to be considered successively.

4.2.1 Unbalanced excitation $\left(\alpha_{1} \ll \alpha_{2}\right.$ with $\left.S_{2} \gg 1\right)$. - The signal intensity is correctly expressed in terms of $\left\langle n_{\mathrm{d}}\right\rangle\left\langle n_{\mathrm{c}}\right\rangle$, (see Eq. (2)) provided $\left\langle n_{\mathrm{d}}\right\rangle \ll\left\langle n_{\mathrm{c}}\right\rangle$, and expressions for those level populations are obtained by solving equations (8) under assumption that $\alpha_{1} \ll \alpha_{2}$. Within the limits of these two conditions, the signal can be considered in two significant limiting cases.

i) Pulse 1 is weak, that is $S_{1} \ll 1$. Then $\left\langle n_{\mathrm{d}}\right\rangle=\frac{N}{4} G^{2} S_{1}$ and it remains much smaller than $\left\langle n_{c}\right\rangle$ for any value of $t_{12}$. The signal is given by :

$$
W\left(t_{12}\right) \propto \frac{N^{2}}{8} G^{2} S_{1}
$$

As a function of delay time it exhibits a dip centered at $t_{12}=0\left[W\left(t_{12}=0\right)=0\right]$, with width of the order of $\tau_{\mathrm{c}}$.

ii) Pulse 1 is strong, that is $S_{1} \gg 1$. Then the condition $\left\langle n_{\mathrm{d}}\right\rangle \ll\left\langle n_{\mathrm{c}}\right\rangle$ is fulfilled only for very small time delays $t_{12}<\tau_{\mathrm{c}} S_{1}^{-1 / 2}$ In this region, the signal varies as :

$$
W\left(t_{12}\right) \propto \frac{N^{2}}{8} \frac{t_{12}^{2}}{\tau_{c}^{2}} S_{1} .
$$

It vanishes at $t_{12}=0$ and then it increases as a function of $\left|t_{12}\right|$. When $\left|t_{12}\right|$ is larger than $\tau_{\mathrm{c}} S_{1}^{-1 / 2}$, the population in state $|\mathrm{d}\rangle$ becomes of the order of the population in the other two states : $\left\langle n_{\mathrm{d}}\right\rangle \simeq\left\langle n_{0}\right\rangle \simeq\left\langle n_{\mathrm{c}}\right\rangle=N / 3$. Then the signal reaches a $t_{12}$-independent value. Thus, for $\alpha_{2} \gg \alpha_{1}$ and $S_{1} \gg 1$, the signal, as a function of the time delay, represents a profound dip of width $\delta t_{12} \cong \tau_{c} S_{1}^{-1 / 2} \ll \tau_{c}$, in agreement with the experimental results. It should be stressed that the atomic evolution characteristic time should not be confused with the width of the $t_{12}$ domain over which the signal intensity varies significantly. The former parameter is given by the inverse interaction rate of the driving fields, $\alpha^{-1}$ Calculations and experiments are performed within the limits of the short correlation-time requirement, $\alpha_{1} \tau_{c} \ll 1$, which guaranties that the atoms do not evolve during the coherence time of the light. This condition is consistent with fast variation of the signal as a function of $t_{12}$, since the width of the signal $t_{12}$-dependent structure is smaller that $\tau_{c}$ as soon as $\alpha_{1} \tau_{\mathrm{L}} \gg 1$.

4.2.2 Balanced excitation by two strong pulses $\left(S_{1}=S_{2} \gg 1\right)$. - The detected quantity, $\left\langle\rho_{12}\right\rangle=\frac{1}{2}\left\langle n_{\mathrm{c}}-n_{\mathrm{d}}\right\rangle$, is correctly represented by equation (10) provided $G \ll 1$. For large enough values of the saturation parameter $S_{1}=S_{2}=S$, that condition is satisfied wherever the expression for $\left\langle\rho_{12}\right\rangle$ significantly departs from 0 . Then, the signal profile, as a function of 
$t_{12}$, exhibits a sharp, Gaussian shaped, peak, located at $t_{12}=0$. The HWHM of this peak is $2 \tau_{\mathrm{c}} \sqrt{\frac{2 \mathrm{Ln} 2}{3 S}}$ It can be much smaller than $\tau_{\mathrm{c}}$, as observed experimentally.

Isolation of the dark state is currently related to the correlation between the driving fields [6]. A parallel can be drawn between correlation and time delay. In the above discussion, the fields $\Omega_{1}$ and $\Omega_{2}$ are assumed to be totally correlated. It can be shown that the same population can be achieved in the dark state either by two time-delayed totally cross-correlated fields or by two synchronous partially cross-correlated fields. The two relevant excitation schemes are related by the following equation :

$$
\left\langle\Omega_{1}^{*}(t) \Omega_{2}(t-\tau)\right\rangle=\sqrt{\alpha_{1} \alpha_{2}} \sqrt{1-G^{2}} g(\tau) / \tau_{\mathrm{c}}
$$

which expresses the cross-correlation function of the synchronous partially cross-correlated fields in the latter scheme in terms of the time delay $t_{12}$ of the totally cross-correlated fields in the former one. This expression can be compared with equation (6) where total correlation is assumed. The factor $\sqrt{1-G^{2}}$ in equation (13) accounts for partial correlation. This factor, which depends on $t_{12}$, departs from unity the more as the fields are less correlated.

We have assumed that the time delay $t_{12}$ is the same for all the illuminated atoms. In the actual experimental situation, where angled beams are used, the time delay at position $r$ is given by :

$$
t_{12}(\mathbf{r})=t_{12}+\left(\mathbf{n}_{2}-\mathbf{n}_{1}\right) \cdot \mathbf{r} / c
$$

where $\mathbf{n}_{1}=\mathbf{k}_{l} / k_{l}$. The beam diameter is $d=3 \mathrm{~mm}$ and the angle $\left(\mathbf{n}_{2}, \mathbf{n}_{1}\right)$ is $2.5 \times 10^{-2} \mathrm{rad}$. Thus, the transverse variation of $t_{12}$ in the interaction region is :

$$
\delta t_{12}=2 \frac{d}{\mathrm{c}} \sin \left(\left(\mathbf{n}_{2}, \mathbf{n}_{1}\right) / 2\right)
$$

which is much smaller than the width of the observed dip in the signal profile. Thus, all the illuminated atoms in the sample evolve in the same manner. This situation is in sharp contrast to one in which counterpropagating pulses are used. Then, according to equation (14), $t_{12}$ strongly depends on the axial position $x$ since $\mathbf{n}_{2}=-\mathbf{n}_{1}$. If $t_{12}=0$ at position $x_{0}$, the atoms with the same final state of excitation as those at $t_{12}=0$ are confined in the region :

$$
\delta x=c \tau_{c}\left(S_{1}\right)^{-1 / 2}
$$

around $x_{0}$. A spatial resolution of order of $1 \mu \mathrm{m}$ can be achieved with realistic values of $\tau_{c}$ and of $S_{1}$. Such an interaction with counterpropagating correlated broadband beams appears to be a promising way to prepare and to monitor atomic particles in a definite internal state at a well defined spatial position. Considerable attention has been paid recently to this problem [13, 14]. In contrast with some previously proposed methods [15], the present approach should enable one to achieve high spatial resolution in the absence of any external potentials characterized by large gradients. In order to detect only those atoms which have been spatially selected, it should be appropriate to record a signal that can be expressed in terms of $\left\langle\rho_{12}\right\rangle$, which, as it is demonstrated above, differs from zero only in the vicinity of $t_{12}=0$. 


\section{Summary.}

The importance of dark resonance in the excitation of a three-level atom by time-delayed stochastic fields has been investigated. The detected coherent transient signals exhibit sharp temporal structures which reflect the time evolution of the first-order statistical moments of the atomic quantities. Stochastic excitation of three-level atoms by correlated counterpropagating pulses is proposed as a method to achieve spatially-selective preparation of those atoms in a definite internal state.

\section{Acknowledgments.}

This research is supported by a C.N.R.S.-N.S.F. International Grant (N.S.F. Grant INT88015036). The work of V.F. and P.B. is also supported by the U.S. Office of Naval Research.

\section{References}

[1] For a review see SHORE B. W., J. Opt. Soc. Am. B 1 (1984) 176.

[2] Tchénio P., Débarre A., Keller J.-C., Le Gouët J.-L., Phys. Rev. A 38 (1988) 5235, Phys. Rev. Lett. 62 (1989) 415, Phys. Rev. A 39 (1989) 1970.

[3] Finkelstein V., Berman P. R., Phys. Rev. A 41 (1990) 6193.

[4] Alzetta G., Gozzini A., Mol L., OrRiols G., Nuovo Cimento 36B (1976) 5 ;

Arimondo E., OrRiols G., Lett. Nuovo Cimento 17 (1976) 333;

Gray H. R., Whitley R. W., Stroud C. R., Opt. Lett. 3 (1978) 218.

[5] Aspect A., Arimondo E., Kaiser R., Vansteenskiste N., Cohen-Tannoudi C., Phys. Rev. Lett. 61 (1988) 826.

[6] Dalton B. J., Knight P. L., J. Phys. B : At. Mol. Phys. 15 (1982) 3997.

[7] Vardeny Z., Tauc J., Opt. Commun. 39 (1981) 396.

[8] IPPEN E. P., ShaNK C. V., in Ultrafast short light Pulses, S. L. Shapiro Ed. (Springer-Verlag, Berlin, 1977) pp. 83-121.

[9] Hong C. K., Ou Z. Y., MANdel L., Phys. Rev. Lett. 59 (1987) 2044.

[10] Finkelstein V., Phys. Rev. A 43 (1991) 4901.

[11] Debarre A., Keller J.-C., Le gouët J.-L., Tchénio P., J. Opt. Soc. Am. B 8 (1991) 153.

[12] VAN KAMPEN N. G., Stochastic processes in Physics and Chemistry, North-Holland.

[13] Balykin V. I., Letokhov V. S., Phys. Today 42 (1989) 23.

[14] Salomon C., Dalibard J., Aspect A., Metcalf H., Cohen-Tannoudi, Phys. Rev. Lett. 59 (1987) 1659.

[15] Thomas J. E., Opt. Lett. 14 (1989) 1186 ; Phys. Rev. A 42 (1990) 5652. 